EPJ Web of Conferences 59, 09004 (2013)

DOI: $10.1051 /$ epjconf/20135909004

(C) Owned by the authors, published by EDP Sciences, 2013

\title{
Beam dynamics analysis in pulse compression using electron beam compact simulator for Heavy Ion Fusion
}

\author{
Takashi Kikuchi ${ }^{1, a}$, Kazuhiko Horioka ${ }^{2}$, Toru Sasaki ${ }^{3}$ and Nob. Harada ${ }^{3}$ \\ ${ }^{1}$ Department of Nuclear System Safety Engineering, Nagaoka University of Technology, \\ 1603-1 Kamitomioka, Nagaoka 940-2188, Japan \\ ${ }^{2}$ Department of Energy Sciences, Tokyo Institute of Technology, 4259 Nagatsuta, Yokohama \\ 226-8502, Japan \\ ${ }^{3}$ Department of Electrical Engineering, Nagaoka University of Technology, 1603-1 \\ Kamitomioka, Nagaoka 940-2188, Japan
}

\begin{abstract}
In a final stage of an accelerator system for heavy ion inertial fusion (HIF), pulse shaping and beam current increase by bunch compression are required for effective pellet implosion. A compact simulator with an electron beam was constructed to understand the beam dynamics. In this study, we investigate theoretically and numerically the beam dynamics for the extreme bunch compression in the final stage of HIF accelerator complex. The theoretical and numerical results implied that the compact experimental device simulates the beam dynamics around the stagnation point for initial low temperature condition.
\end{abstract}

\section{INTRODUCTION}

Transport of space charge dominated beams with low emittance is crucial issue for application to inertial confinement fusion (ICF) driven by heavy ion beams [1,2]. A compact simulator with an electron beam was constructed to understand beam dynamics during final pulse compression for ICF driven by heavy ion beams $[3,4]$. It is important to clear the beam dynamics for the precise control of high-current charged particle beams due to effective fuel pellet implosion of ICF. To investigate the limitation of longitudinal pulse compression, we study the space-charge dominated beam dynamics with theoretical and numerical simulation approaches [5].

\section{THEORETICAL ESTIMATION TO CREATE SPACE CHARGE DOMINATED BEAMS}

Using a longitudinal beam envelope equation, the ratio between the repulsion forces due to the space charge and the emittance of the beam bunch is estimated. The envelope equation is written by [6]

$$
z_{m}^{\prime \prime}+k_{\|} z_{m}-\frac{K_{\|}}{z_{m}^{2}}-\frac{\varepsilon_{\|}^{2}}{z_{m}^{3}}=0
$$

where $z_{m}$ is the half bunch length, $K_{\|}$is the longitudinal perveance, $k_{\|}$is the longitudinal focusing force, $\epsilon_{\|}$is the longitudinal emittance, respectively. From third and fourth terms in the left hand side of Eq. (1), the condition of the space charge dominated beam is obtained by

$$
\frac{K_{\|} z_{m}}{\varepsilon_{\|}^{2}}=\frac{3 e g I_{b 0}}{20 \pi \epsilon_{0} v_{z} k_{B} T_{\|}} \frac{z_{m 0}}{z_{m}}>1,
$$

\footnotetext{
${ }^{a}$ e-mail: tkikuchi@vos.nagaokaut.ac.jp
}

This is an Open Access article distributed under the terms of the Creative Commons Attribution License 2.0, which permits unrestricted use, distribution, and reproduction in any medium, provided the original work is properly cited. 




Figure 1. Ratio of longitudinal repulsion force due to space charge and emittance at each temperature.

where $e$ is the elementary charge, $g$ is the geometry factor, $I_{b 0}$ is the initial beam current, $\tau_{b 0}$ is the initial pulse duration, $\epsilon_{0}$ is the permittivity in vacuum, $k_{B} T_{\|}$is the longitudinal temperature, $k_{B}$ is the Boltzmann constant, respectively.

The estimation result normalized by the initial bunch length $z_{m 0}$ is shown in Fig. 1. The initial beam current $I_{b 0}$ is $100 \mu \mathrm{A}$ and the initial beam pulse duration $\tau_{b 0}$ is $100 \mathrm{~ns}$. As a result, it is predicted that the compression ratio of 57.8 is required to demonstrate the space charge dominated beams in the apparatus for $k_{B} T_{\|}=1 \mathrm{eV}$.

\section{NUMERICAL SIMULATION FOR BUNCH COMPRESSION}

Numerical simulations based on a particle-in-cell (PIC) code [7] are carried out. The model is a one-dimensional electrostatic PIC code with the long wave approximation for the longitudinal space charge calculation [6]. Figure 2 shows the computational region for electron beam transport with pulse compression by applying modulation voltage at the gap.

The longitudinal self-electric field $E_{z}$ is calculated by $E_{z}=-\left\{g /\left(4 \pi \epsilon_{0}\right)\right\} d \lambda / d z$ [6], where $\lambda$ is the line-charge density.

The electron bunch is extracted from an electron gun assisted by thermal electron emission. The electron bunch is modulated by the applied electric field in the gap. The voltage pulses produced at each module are overlapped at the gap using induction modulator technology. The overlapped voltage pulse $V_{g}$ at the gap is given by

$$
V_{g}=\frac{m_{e}}{2 e\left\{\sqrt{m_{e} / 2 e V_{0}}+\left(\tau_{V}-t\right) / L\right\}^{2}}-V_{0},
$$




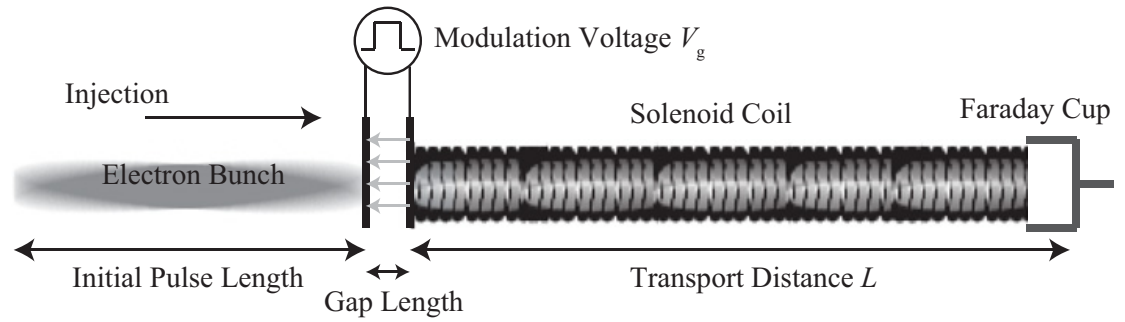

Figure 2. Calculation box of longitudinal 1D PIC simulation for electron bunch compression with transverse confinement due to solenoid coil.



Figure 3. Beam currents normalized by initial one without space charge effect for $k_{B} T_{\|}=0$ at each gap length for (a) $5 \mathrm{~mm}$, (b) $10 \mathrm{~mm}$, (c) $15 \mathrm{~mm}$, (d) $20 \mathrm{~mm}$, and (e) $25 \mathrm{~mm}$, respectively.

where $m_{e}$ is the electron mass, $V_{0}$ is the extraction voltage of the electron beam at the electron gun, $\tau_{V}$ is the pulse duration of the applied voltage, $L$ is the beam transport distance with solenoid magnetic field, respectively. In this study, $V_{0}=2.8 \mathrm{kV}, \tau_{V}=100 \mathrm{~ns}, L=2 \mathrm{~m}$ are assumed to correspond with the experimental condition. By the applied voltage as given by Eq. (3), the head of the beam bunch is decelerated, while the tail of the beam bunch is not modulated. As a result, the beam pulse is compressed during the transport. It is assumed that the extracted electrons are thermalized at the electron gun and mismatch injection into the gap as $v_{t h}=\sqrt{k_{B} T_{\|} / m_{e}}$, where $v_{t h}$ is the initial thermal velocity.

Figure 3 shows the calculation results of the beam current normalized by the initial one at each gap length, i.e., the pulse compression ratio. The initial beam current $I_{b 0}$ is $100 \mu \mathrm{A}$, and the initial beam pulse duration $\tau_{b 0}$ is $100 \mathrm{~ns}$. The applied voltage waveform was assumed by Eq. (3). As shown in 


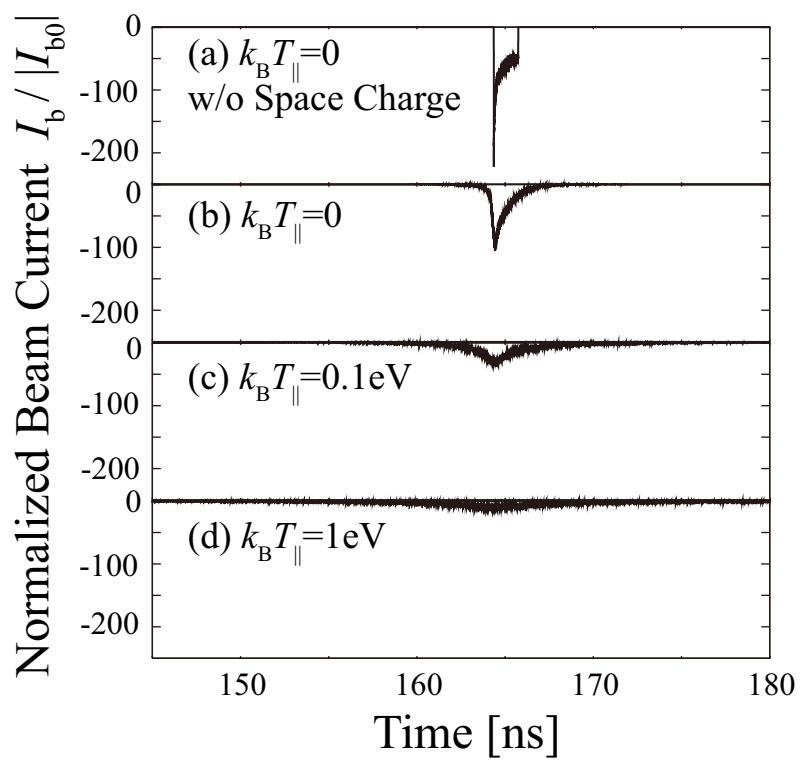

Figure 4. Beam currents normalized by initial one (the gap length is $15 \mathrm{~mm}$ ), for (a) $k_{B} T_{\|}=0$ without space charge effect, (b) $k_{B} T_{\|}=0$ with space charge effect, (c) $k_{B} T_{\|}=0.1 \mathrm{eV}$ with space charge effect, (d) $k_{B} T_{\|}=1 \mathrm{eV}$ with space charge effect, respectively.

Fig. 3, the compressed beam current depends on the gap length. Because the applied voltage given by Eq. (3) is designed by the infinitesimal gap length. As a result, smaller gap length causes the high bunch compression ratio.

Figure 4 shows the calculation results of the beam current normalized by the initial one with and without space charge effect at each initial temperature. The gap length is set as $15 \mathrm{~mm}$. The initial beam current $I_{b 0}$ is $100 \mu \mathrm{A}$, and the initial beam pulse duration $\tau_{b 0}$ is $100 \mathrm{~ns}$. The space charge effect and the initial temperature of electron bunch interfere the extreme pulse compression. In this condition, it is found that the initial temperature of electron bunch is main issue of interference for the extreme pulse compression. In comparisons with Fig. 1, although the compressed electron bunch becomes the space charge dominated state for the case of $k_{B} T_{\|}=0.1 \mathrm{eV}$, the electron bunch is in the condition of emittance dominated regime for $k_{B} T_{\|}=1 \mathrm{eV}$.

\section{CONCLUSION}

In the final bunch compression for heavy ion-beam driven ICF, we studied the beam dynamics with theoretical and numerical simulation approaches to investigate the limitation of longitudinal pulse compression. Using the longitudinal envelope equation, the ratio between the repulsion forces due to the space charge and the emittance was estimated. The numerical simulations were carried out in the parameters of compact electron beam experimental device. These theoretical and numerical approaches suggested that if the initial temperature is low enough, the compact simulator will be able to simulate the beam dynamics around the stagnation point at the unneutralized bunch compression.

\section{References}

[1] K. Horioka, et al., Nucl. Instrum. Methods Phys. Res. A606, 1 (2009), and references therein

[2] A. Friedman, et al., Nucl. Instrum. Methods Phys. Res. A606, 6 (2009), and references therein 


\section{IFSA 2011}

[3] K. Horioka, et al., 18th International Symposium on Heavy Ion Inertial Fusion (HIF2010), Darmstadt, August-September 2010, TUS-0404

[4] A. Nakayama, et al., "Longitudinal bunch compression study with induction voltage modulator", these proceedings

[5] A. Namprom, T. Wiboonphon, T. Sasaki, and T. Kikuchi, Nob. Harada, 3rd Euro-Asian Pulsed Power Conference / 18th International Conference on High-Power Particle Beams EAPPCBEAMS 2010, Jeju, Korea, October 10-14, 2010, MoB3-5, p.108

[6] M. Reiser, Theory and Design of Charged Particle Beams (Wiley, New York, 1994)

[7] R. W. Hockney and J. W. Eastwood, Computer Simulation using Particles (IOP publishing, Bristol and Philadelphia, 1988) 